\title{
Uma decomposição genética para o objeto matemático limite de uma função
}

A genetic decomposition for the mathematical object limit of a function

\author{
Maria Alice de Vasconcelos Feio Messias ${ }^{1}$ \\ João Cláudio Brandemberg ${ }^{2}$
}

\section{Resumo}

O estudo descrito nesse trabalho, o qual apresenta parte dos resultados de uma pesquisa de doutorado, teve o objetivo de conjecturar sobre que estruturas e mecanismos mentais precisam ser construídos por um indivíduo, de maneira a possibilitá-lo compreender o conceito de limite de uma função. Baseamo-nos, desse modo, nos pressupostos da teoria APOS (DUBINSKY et al., 1984; ARNOON et al., 2014), em nossos conhecimentos sobre o próprio objeto matemático, e em compreensões de estudantes sobre limites, para a formulação de uma Decomposição Genética (DG) para o referido conceito. Nesse sentido, evidenciamos importância de que diferentes objetos matemáticos, tais como o conceito de função, a definição de limite, a relação entre $\varepsilon$ e $\delta$, a relação entre limites laterais e bilateral, as propriedades de limite, limites envolvendo infinito, dentre outros elementos, sejam contemplados em uma DG para o objeto Limite de uma Função. Reiteramos que a Decomposição Genética apresentada poderá nortear tanto instrumentos avaliativos quanto materiais instrucionais que viabilizem o processo de aprendizagem no âmbito do Cálculo.

Palavras chave: limite de uma função; decomposição genética; teoria APOS.

\section{Abstract}

The study presented in this paper, which shows part of the results of a doctoral research, has the aim of conjecture about mental structures and mechanisms need to be built by an individual, in order to enable the comprehension of the limit concept. We were based on the assumptions of APOS theory (DUBINSKY et al., 1984; ARNOON et al., 2014), in what we know about such mathematical object, besides students comprehensions about limits, to create a Genetic Decomposition (GD) to the referred concept. In this sense, we noticed the importance of different mathematical objects, such as function, limit definition, $\varepsilon-\delta$ relation, lateral and bilateral limits, limits properties, limits involving infinity, above others, as part of a GD for the object Limit of a Function. We reiterate that such Genetic Decomposition may guide both, evaluative instruments and instructional materials in order to make the learning process in Calculus possible.

Keywords: limit of a function; genetic decomposition; APOS theory.

\footnotetext{
${ }^{1}$ Universidade do Estado do Pará; Universidade da Amazônia | alice.messias@gmail.com

${ }^{2}$ Universidade Federal do Pará | brand@ufpa.br
} 


\section{Introdução}

A apreensão de conceitos matemáticos se apresenta como um processo que não depende, simplesmente, da memorização de definições e/ou de habilidades operatórias específicas. Consideramos, desse modo, as ações interiorizadas por um indivíduo sobre determinado conceito e, principalmente, os significados dados a essas ações, fundamentais para a compreensão de qualquer conhecimento matemático (MESSIAS, 2018).

Nessa perspectiva, admitimos que a aprendizagem matemática esteja condicionada a um amplo conjunto de interações inerentes à sua própria composição. Assumimos, portanto, que para efetivamente compreender um conceito, é preciso refletir sobre ele, e buscar nele (e em diferentes contextos) a abstração por meio de suas representações, generalizações e sintetizações.

Ressaltamos, ainda, que a execução de tarefas, sem refletir, ou mesmo entender os conceitos nela envolvidos, prejudica a compreensão de ideias matemáticas, e o consequente amadurecimento de construções mentais que contribuem para a formação de um pensamento matematicamente maduro e coerente com suas representações teóricas.

Frente a tais considerações, e diante das experiências que vivenciamos na docência em Cálculo, aprofundamos nossos estudos no que se refere à compreensão de estudantes acerca dos elementos que constituem essa área de conhecimento, de modo que as expressivas dificuldades inerentes ao entendimento sobre limite ${ }^{3}$, conforme apontado em diferentes estudos (TALL; VINNER, 1981; KARATAS et al., 2011; AMATANGELO, 2013), motivou-nos a delimitar o campo de ação do estudo apresentado nesse trabalho à problemática da aprendizagem do conceito de limite de uma função.

É importante ressaltarmos que o conceito de limite é de fundamental importância para a construção de uma base mais sólida da esfera de conhecimentos do Cálculo. O teorema do valor médio, por exemplo, é aplicável somente para funções contínuas. Sendo assim, antes de utilizá-lo, é preciso averiguar a continuidade da função em um dado intervalo ou domínio e, para tanto, verificar as condições para a existência do limite em determinado ponto. Qualquer fragilidade no que tange ao entendimento do conceito de limite pode implicar, portanto, em dificuldades na aprendizagem de outros conteúdos no âmbito do Cálculo.

Colocamo-nos, então, diante de uma pergunta: Que estruturas e mecanismos mentais precisam ser construídos de modo a permitir que estudantes compreendam o conceito de limite de uma função? Para respondê-la, desenvolvemos uma pesquisa ${ }^{4}$ - à luz da Teoria APOS (DUBINSKY et al., 1984; ARNOON et al., 2014) - que teve como um de seus objetivos, conjecturar sobre que estruturas e mecanismos mentais precisam ser construídos por um indivíduo de modo a possibilitá-lo compreender efetivamente o conceito de limite.

Na perspectiva da pergunta e objetivo enunciados, apresentamos nesse trabalho uma Decomposição Genética (DG) para o objeto matemático Limite de uma Função. Para tanto e com o intuito de viabilizar o entendimento acerca do processo de construção da DG traçamos uma reflexão sobre múltiplas compreensões de estudantes de graduação, relativas ao conceito de limite (MESSIAS, 2018). Em seguida, discutimos a Teoria APOS (DUBINSKY et

\footnotetext{
${ }^{3}$ Os termos 'limite' e 'limite de uma função' são utilizados indistintamente no decorrer desse artigo.

${ }^{4}$ Referimo-nos à pesquisa de doutorado da primeira autora.
} 
al., 1984; ARNOON et al., 2014), relacionando-a, nos tópicos subsequentes, com o estudo realizado, seus aspectos metodológicos e, claro, com a DG elaborada. Em nossas considerações finais, discutimos a relevância e limitações desse trabalho, bem como nossas expectativas para com suas contribuições para pesquisas nas áreas de Educação em Ciências e Matemáticas e Educação Matemática no Brasil.

\section{Compreensões sobre o conceito de Limite de uma Função}

A aprendizagem de conceitos matemáticos no âmbito do Cálculo têm sido objeto de estudo em várias pesquisas nacionais e internacionais. Dedicamos esse tópico à discussão relativa aos múltiplos conhecimentos de estudantes no que tange ao conceito de limite. Para tanto, norteamo-nos em compreensões sobre do objeto Limite de uma função evocadas por sujeitos investigados na pesquisa de doutorado da primeira autora desse artigo (MESSIAS, 2018), as quais destacamos no decorrer desse tópico.

[C1] - A existência do limite depende dos limites laterais;

[C2] - $\lim _{x \rightarrow x_{0}} f(x)$ existe se $\lim _{x \rightarrow x_{0}^{+}} f(x)$ e $\lim _{x \rightarrow x_{0}^{-}} f(x)$ existem e são iguais e se $\lim _{x \rightarrow x_{0}} f(x)=f\left(x_{0}\right)$;

[C3] - $\lim _{x \rightarrow x_{0}} f(x)$ existe se $x_{0} \in D_{f}$;

[C4] - 'Saltos' implicam em limites laterais diferentes e, consequentemente, na não existência do limite;

[C5] - O limite representa uma aproximação no eixo das abscissas;

[C6] - $x \rightarrow x_{0}^{+}$e $x \rightarrow x_{0}^{-}$implica que $f(x) \rightarrow L$;

[C7] - O limite é uma aproximação em torno/à direita e à esquerda de $x_{0}$ que implica em uma aproximação de $f$ em relação a um valor de $y$ correspondente;

[C8] - Teorema do confronto: $L$ existe quando $x \rightarrow x_{0}$ se os limites laterais existem e estes são iguais;

[C9] - Para calcular o limite de uma função escrita em partes quando $x \rightarrow x_{0}$, basta fazer $x=x_{0}$ em todas as equações que compõem $f(x)$;

[C10] - L é o correspondente em $y$ para $x=x_{0} ; f\left(x_{0}\right)=L$;

[C11] $-\lim _{x \rightarrow x_{0}} f(x)$ significa que quando $x \rightarrow x_{0}$ pela direita e pela esquerda $f(x) \rightarrow$ $L ;$

[C12] - $\lim _{x \rightarrow x_{0}} f(x)$ será sempre igual a $f\left(x_{0}\right)$;

[C13] - $L$ e o comprimento de uma reta;

No que concerne às compreensões [C1] e [C2], observamos que Messias (2018) identificou que muitos estudantes relacionam de maneira coerente a existência do limite bilateral com seus limites laterais. Porém, algumas evocações, como aquelas que caracterizaram as compreensões [C3] e [C4], são pautadas em interpretações equivocadas da natureza do conceito de limite.

No caso de [C3], a existência de $\lim _{x \rightarrow x_{0}} f(x)$ é condicionada a $x_{0} \in D_{f}$. Esse tipo de interpretação já fora discutido em outros estudos (PRZENIOSLO, 2004; JORDAAN, 2005; KARATAS et al., 2011; DENBEL, 2014; MESSIAS; BRANDEMBERG, 2015). Os objetos Limite e Domínio podem, em conjunto, representar um fator de conflito cognitivo, especialmente, se o indivíduo generalizar tal relação a partir do cálculo de limites de funções contínuas, fato que, inclusive, pode levar às compreensões [C10] e [C12], sendo que nessa última, o $\lim _{x \rightarrow x_{0}} f(x)$ é considerado sempre igual a $f\left(x_{0}\right)$ (limite alcançável). Tal ideia foi, também, 
identificada nos trabalhos de Cornu (1991), Hitt e Lara-Chaves (1999), Przenioslo (2004), Juter (2008) Amatangelo (2013).

As compreensões [C5], [C6] e [C7] e [C11] estão, sobretudo, pautadas em interpretações dinâmicas do conceito de limite, em que é atribuído 'movimento' a função (TALL; VINNER, 1981; PRZENIOSLO, 2004; SARVESTANI, 2011; AMATANGELO, 2013), a partir da ideia de aproximação em torno de $x_{0}$ e/ou expressões como 'tende a', 'se aproxima de', dentre outras, que podem reforçar uma percepção tanto de limite inalcançável, isto é, que o valor de $f$ em determinado ponto sempre difere do valor de L, isto é, $\lim _{x \rightarrow x_{0}} f(x) \neq f\left(x_{0}\right)$ (TALL; VINNER, 1981; JUTER, 2008; AMATANGELO, 2013; MESSIAS; BRANDEMBERG, 2015) quanto de limite intransponível, ou seja, como se funcionasse como uma barreira que não pode ser ultrapassada (CORNU, 1983; CORNU, 1991; JUTER, 2008; AMATANGELO, 2013).

Em Messias (2018) também foram identificadas as compreensões [C8] e [C13], as quais reiteram o fato de estudantes apresentarem interpretações equivocadas acerca da natureza do conceito de limite. No caso de [C8], o teorema do confronto foi atrelado aos limites laterais, de modo que estudantes consideraram que a forma algébrica da função definida para os valores em torno de $x_{0}$ (seu domínio de interesse) estaria limitada superiormente e inferiormente por duas funções que convergiam para um mesmo valor limite. Ou seja, ele enxergou as formas algébricas de uma função escrita em partes como diferentes funções que atendiam ao que é estabelecido no referido teorema.

Ressaltamos que tais compreensões sobre o conceito de limite de uma função mostram-se, sobretudo, pautadas em interpretações dinâmicas, especialmente, quando atreladas à percepção de aproximação em torno de um valor tanto no eixo das abscissas quanto no eixo das ordenadas. A existência do limite condicionada ao domínio, à continuidade da função, à ausência de 'saltos' e/ou 'buracos' reforça, também, que a existência do limite tem se configurado como um fator de conflito cognitivo para os estudantes de Cálculo em geral.

Reiteramos que as compreensões destacadas no decorrer desse tópico foram de grande relevância no sentido de nos permitirem verificar possíveis conflitos que permeiam as imagens conceituais de estudantes de Cálculo no que tange ao conceito de limite. A seguir, traçamos algumas considerações sobre a teoria APOS para que, enfim, possamos apresentar uma decomposição genética para o referido objeto matemático.

\section{Considerações sobre a Teoria APOS}

A teoria APOS (Action, Process, Object, Schema) é um quadro teórico utilizado para explicar como indivíduos constroem mentalmente seu entendimento sobre determinado objeto matemático. Nessa perspectiva, essa teoria traz consigo a contextualização de mecanismos mentais, tais como a interiorização, coordenação, reversão, encapsulação, generalização, e tematização, para o contexto do Pensamento Matemático Avançado, com o intuito de possibilitar a construção, por parte de um indivíduo, de estruturas mentais relacionadas a um conceito, por meio de Ações, Processos, Objetos e Esquemas.

Quando um sujeito é confrontado com um contexto matemático específico, ele evoca um Esquema e faz uso de seus componentes para, por exemplo, mediar o processo de aquisição de um novo conhecimento ou solucionar uma tarefa proposta. Nesse sentido, os conceitos matemáticos são primeiramente concebidos por meio de Ações sobre um ou 
mais Objetos pré-existentes. Ao serem interiorizadas, as Ações formam Processos que, por sua vez, são encapsulados em forma de Objetos que podem ser desencapsulados de volta ao Processo pelo qual foram concebidos. São as Ações, Processos e Objetos que compõem um Esquema (MESSIAS, 2018).

Figura 1 - Composição de um Esquema

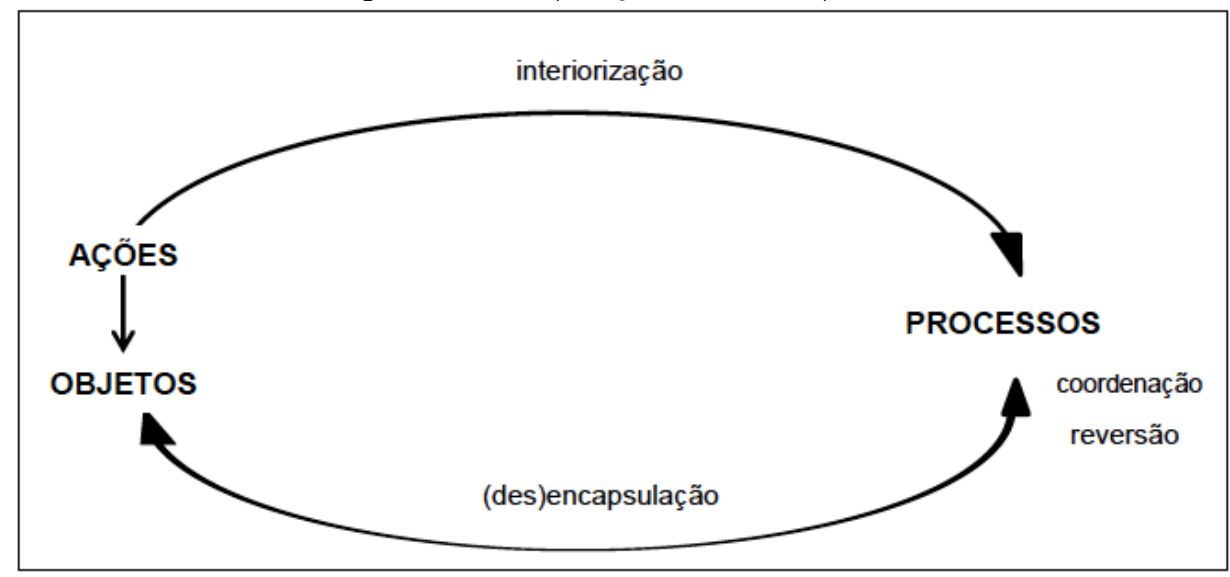

Fonte: Arnon et al. (2014, p. 18, traduzido pela autora)

Mediante a figura 1, compreendemos que seja a partir da manipulação de objetos físicos ou mentais previamente existentes que um indivíduo formula Ações. À medida que este tem controle sobre suas Ações, elas são interiorizadas como Processo. É nesse momento em que passa a ter consciência acerca dessas Ações, fato que o possibilita refletir sobre elas e combiná-las entre si e, inclusive, entre outras Ações.

Quando o indivíduo consegue entender um Processo como uma estrutura estática, de maneira a realizar Ações sobre tal estrutura, dizemos que este alcançou a Encapsulação, fato que o permite atribuir ao referido processo o status de Objeto. Muitos processos cognitivos estão normalmente envolvidos nessa construção, sendo que um ou mais Objetos podem ser desencapsulados e seus respectivos Processos, Coordenados de maneira a formar um novo Objeto.

Um Esquema sobre um objeto matemático é dinâmico; sua coerência é determinada pela habilidade que um indivíduo tem, ao verificar em quais situações matemáticas deve evocá-lo e, consequentemente, sua coleção de Ações, Processos, Objetos e, inclusive, de outros Esquemas (MESSIAS, 2018). Essa estrutura mental pode diferir de um indivíduo para outro, já que uma multiplicidade de relações entre seus componentes pode ser construída sem, necessariamente serem evocados sempre que uma situação envolva uma mesma ideia matemática. Isso porque, o aprendizado matemático não se desenvolve de maneira linear (ARNON et al., 2014).

Ressaltamos que um Esquema pode ser uma importante ferramenta para entender como o conhecimento matemático é estruturado na mente do indivíduo, já que é possível prever as condições para o aprendizado de um conceito a partir de modelos que conjecturem sobre suas construções mentais (MESSIAS, 2018). Nessa perspectiva, a teoria aponta para a determinação de um modelo hipotético que descreve estruturas e mecanismos mentais que um indivíduo pode precisar construir para que compreenda um conceito matemático específico. Tal modelo recebe o nome de Decomposição Genética (ARNON et al., 2014). 
Uma Decomposição Genética (DG) é, em primeiro lugar, uma hipótese. Para sua formulação inicial, devemos nos nortear em experiências prévias relacionadas ao ensino e aprendizagem do conceito matemático em questão, bem como no próprio conhecimento que temos acerca desse conteúdo e de seu desenvolvimento histórico conceitual. Um entendimento sólido sobre a teoria APOS, e a efetivação de uma revisão bibliográfica que contemple estudos relativos à compreensão de estudantes acerca da temática escolhida podem, também, contribuir para a elaboração de decomposições genéticas (ARNON et al., 2014).

Em acordo com MESSIAS (2018), o referido modelo contempla uma descrição das 'transformações' que um sujeito precisa realizar em um Objeto mental pré-existente para que, a partir delas, seja possível construir um novo conceito matemático. Isto é, uma decomposição genética pode nos permitir conjecturar sobre como Ações, Processos e Objetos podem ser organizados para formar Esquemas coerentes que viabilizem a apreensão de um conceito. Nesse sentido, Roa-Fuentes e Oktaç (2010) apontam que uma DG se apresenta como um modelo de epistemologia e cognição matemática.

É relevante afiançarmos que uma decomposição genética não é necessariamente única, haja vista que o entendimento de um conceito não acontece da mesma maneira para todos os indivíduos. Todavia, podemos utilizá-la como um modelo geral que descreve trajetórias para a construção de um conceito. Ela serve, ainda, como um instrumento diagnóstico que, por sua vez, pode auxiliar na elaboração de instruções de ensino que contemplem os possíveis caminhos cognitivos para a aquisição de um determinado conceito matemático.

Finalmente, ressaltamos que seja possível conjecturar, a partir de uma decomposição genética, acerca dos mecanismos e estruturas mentais que julgamos ser necessários para a compreensão de determinado conhecimento matemático, fato que nos levou à efetivação do estudo, o qual temos o intuito de apresentar nesse trabalho e, cujos aspectos metodológicos são apresentados no tópico subsequente.

\section{Delimitação do estudo realizado}

O estudo apresentado nesse artigo se constituiu como parte da pesquisa de doutoramento da primeira autora ${ }^{5}$. Nesse texto, restringimo-nos ao objetivo de conjecturar sobre que estruturas e mecanismos mentais precisam ser construídos por um indivíduo de modo a possibilitá-lo compreender efetivamente o conceito de limite. Tal objetivo foi norteado pela seguinte pergunta: Que estruturas e mecanismos mentais precisam ser construídos de modo a permitir que estudantes compreendam o conceito de limite de uma função?

Para responder a referida pergunta e, consequentemente, alcançar o objetivo estabelecido, efetivamos um estudo que nos possibilitou construir uma decomposição genética para Limite de uma Função. Consideramos, para essa construção, nossas experiências docentes no âmbito do Cálculo, nosso entendimento acerca da teoria APOS, além das treze compreensões sobre limite elencadas anteriormente nesse artigo (ver item

\footnotetext{
${ }^{5}$ Para maiores esclarecimentos, sugerimos a leitura de Messias (2018).
} 
2). Alguns aspectos inerentes ao desenvolvimento histórico desse conceito também foram considerados.

No tópico subsequente, traçamos algumas considerações prévias frente aos aspectos que nos nortearam a definir os elementos contemplados na decomposição genética elaborada.

\section{Decomposição genética (DG) para o objeto Limite de uma função}

Para a elaboração da DG tomamos, inicialmente, as compreensões [C1] a [C13], destacadas no item 2 desse artigo, de modo que as aglutinamos quanto à natureza do conceito do limite, (não) existência do limite e outras compreensões, conforme destacado a seguir.

(i) Quanto à natureza do conceito de limite

Incluímos nesse grupo as seguintes compreensões: [C5] O limite representa uma aproximação no eixo das abscissas; [C6] $x \rightarrow x_{0}^{+}$e $x \rightarrow x_{0}^{-}$implica que $f(x) \rightarrow L$; [C7] $O$ limite é uma aproximação em torno/à direita e à esquerda de $x_{0}$ que implica em uma aproximação de $f$ em relação a um valor de $y$ correspondente; [C10] $L$ é o correspondente em $y$ para $x=x_{0} ; f\left(x_{0}\right)=L_{\text {; }}$ [C11] $\lim _{x \rightarrow x_{0}} f(x)$ significa que quando $x \rightarrow x_{0}$ pela direita $\mathrm{e}$ pela esquerda $f(x) \rightarrow L$; [C12] $\lim _{x \rightarrow x_{0}} f(x)$ será sempre igual a $f\left(x_{0}\right)$ e [C13] $L$ é o comprimento de uma reta.

As compreensões [C5], [C6], [C7] e [C11] estão vinculadas à ideia de aproximação em torno de um ponto, sendo que [C5] pode se constituir como um fator de conflito em potencial, uma vez que restringe essa aproximação ao eixo das abscissas. Ponderamos, nesse sentido, que os seguintes aspectos podem ser considerados no decorrer de apreensão do conceito de limite: Avaliar uma função $f(x)$ em sucessivos pontos em torno de $x_{0}$; avaliar uma função $f$ em sucessivos pontos em torno de $f\left(x_{0}\right)$; relacionar entre si os sucessivos pontos em torno de $x_{0}$ e $f\left(x_{0}\right)$.

Admitimos que as compreensões [C10] e [C12] estejam, possivelmente, vinculadas à prática excessiva do cálculo de limite de funções contínuas. É comum, nesse sentido, que muitos estudantes não consigam diferenciar $\lim _{x \rightarrow x_{0}} f(x)$ e $f\left(x_{0}\right)$. Entendemos, portanto, que tal prática precisa ser diversificada, de modo a: relacionar o $\lim _{x \rightarrow x_{0}} f(x)$ e $f\left(x_{0}\right)$ de diferentes funções para que o sujeito perceba que nem sempre $\lim _{x \rightarrow x_{0}} f(x)=f\left(x_{0}\right)$; solicitar que o aluno interprete limites a partir de diferentes representações.

Finalmente, a compreensão evocada em [C13] nos chamou atenção para a importância de estabelecer uma discussão frente às múltiplas interpretações e representações de limite.

(ii) Quanto à (não) existência do limite

Incluímos nesse grupo as quatro compreensões, a saber: [C1] A existência do limite depende dos limites laterais; [C2] $\lim _{x \rightarrow x_{0}} f(x)$ existe se $\lim _{x \rightarrow x_{0}^{+}} f(x)$ e $\lim _{x \rightarrow x_{0}^{-}} f(x)$ existem e são iguais e se $\lim _{x \rightarrow x_{0}} f(x)=f\left(x_{0}\right)$; [C3] $\lim _{x \rightarrow x_{0}} f(x)$ existe se $x_{0} \in D_{f}$; [C4] Saltos implicam em limites laterais diferentes e, consequentemente, na não existência do limite; [C8] Teorema do confronto: $L$ existe quando $x \rightarrow x_{0}$ se os limites laterais existem $\mathrm{e}$ estes são iguais; 
Evidenciamos, mediante tais compreensões, que as imagens conceituais dos sujeitos investigados foram constituídas, principalmente, por evocações relacionadas aos limites laterais. Reiteramos, nesse sentido que: Seja preciso avaliar a (não) existência do limite em diferentes situações e, a partir de múltiplas representações; O teorema do confronto, e sua relação com a existência do limite de uma função $f$, cujo domínio de interesse é limitado por duas funções que convergem para o mesmo limite, devem ser discutidos por meio de práticas que enfatizem essa relação; "O que os 'saltos' no gráfico da função representam?" Esse questionamento deve ser levantado, pois, em geral, é vinculado a interpretações incoerentes sobre a relação entre domínio, limite e continuidade.

A compreensão evocada em [C3] tem constituído parte da imagem conceitual de muitos estudantes de Cálculo. Entendemos, nesse sentido, que: Mais uma vez, a prática excessiva do cálculo de limite de funções contínuas pode levar a esse tipo de compreensão; A interpretação da definição de limite sob o ponto de vista geométrico pode trazer esclarecimentos sobre a incoerência de tal compreensão.

\section{(iii) Outras compreensões}

Foi incluída nesse grupo a compreensão [C9] Para calcular o limite de uma função escrita em partes quando $x \rightarrow x_{0}$, basta fazer $x=x_{0}$ em todas as equações que compõem $f(x)$. Evidenciamos, desse modo, que o cálculo de limites de funções escritas em mais de uma sentença tem se configurado como um fator de conflito em potencial. Nesse sentido: Reflexões do tipo 'existe diferença entre $x \rightarrow x_{0}$ e $x=x_{0}$ ?' precisam ser levantadas no decorrer do processo de apreensão do conceito de limite; O cálculo de limite de uma função escrita em partes precisa contemplar reflexões relativas às suas formas algébrica e geométrica, domínio e imagem.

A partir de nossas considerações prévias acerca das compreensões sobre o conceito de limite, elencadas nesse artigo, elaboramos um esboço da decomposição genética, a qual é constituída por múltiplas construções mentais.

Apresentamos nas figuras 2 a 7, distribuídas no tópico seguinte, conjecturas acerca de construções mentais relativas ao conceito de limite de uma função (que julgamos ser) necessárias para compor a decomposição genética prevista na pesquisa.

\section{Uma decomposição genética para limite de uma função}

A DG elaborada é constituída por 6 esquemas integrados. O primeiro Esquema é constituído de mecanismos e estruturas mentais que julgamos necessários para a construção do objeto definição de limite de uma função (ver figura 2).

No que concerne às Ações [A1] e [A2], admitimos que um sujeito precise incialmente, avaliar diferentes funções em sucessivos pontos em torno de $x_{0}$ e de $f\left(x_{0}\right)$ (exceto talvez em $x_{0}$ e $f\left(x_{0}\right)$ ), relacionando-os de maneira que seja possível alcançar a compreensão da linguagem matemática $x \rightarrow x_{0}$ e $f(x) \rightarrow L$. O termo 'Limite' também pode ser introduzido ao sujeito. Ressaltamos, nesse sentido, a importância de tais Ações serem realizadas mediante múltiplas representações de funções (forma algébrica, gráfico, tabela).

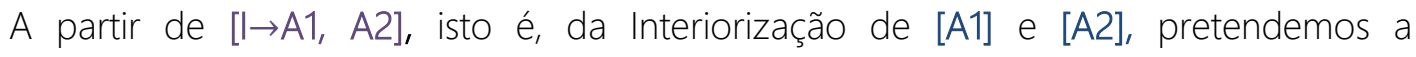
construção de uma relação de pares ordenados entre os elementos pertencentes aos intervalos $(x-\delta, x+\delta)$ e $(L-\varepsilon, L+\varepsilon)$. Para tanto, consideramos coerente que o sujeito parta da representação gráfica de uma determinada função, de modo que ele seja levado a 
generalizá-la. É importante que o indivíduo consiga compreender o que $\varepsilon$ e $\delta$ representam em seus respectivos intervalos.

Figura 2 - Esquema 1: Definição de Limite de uma Função

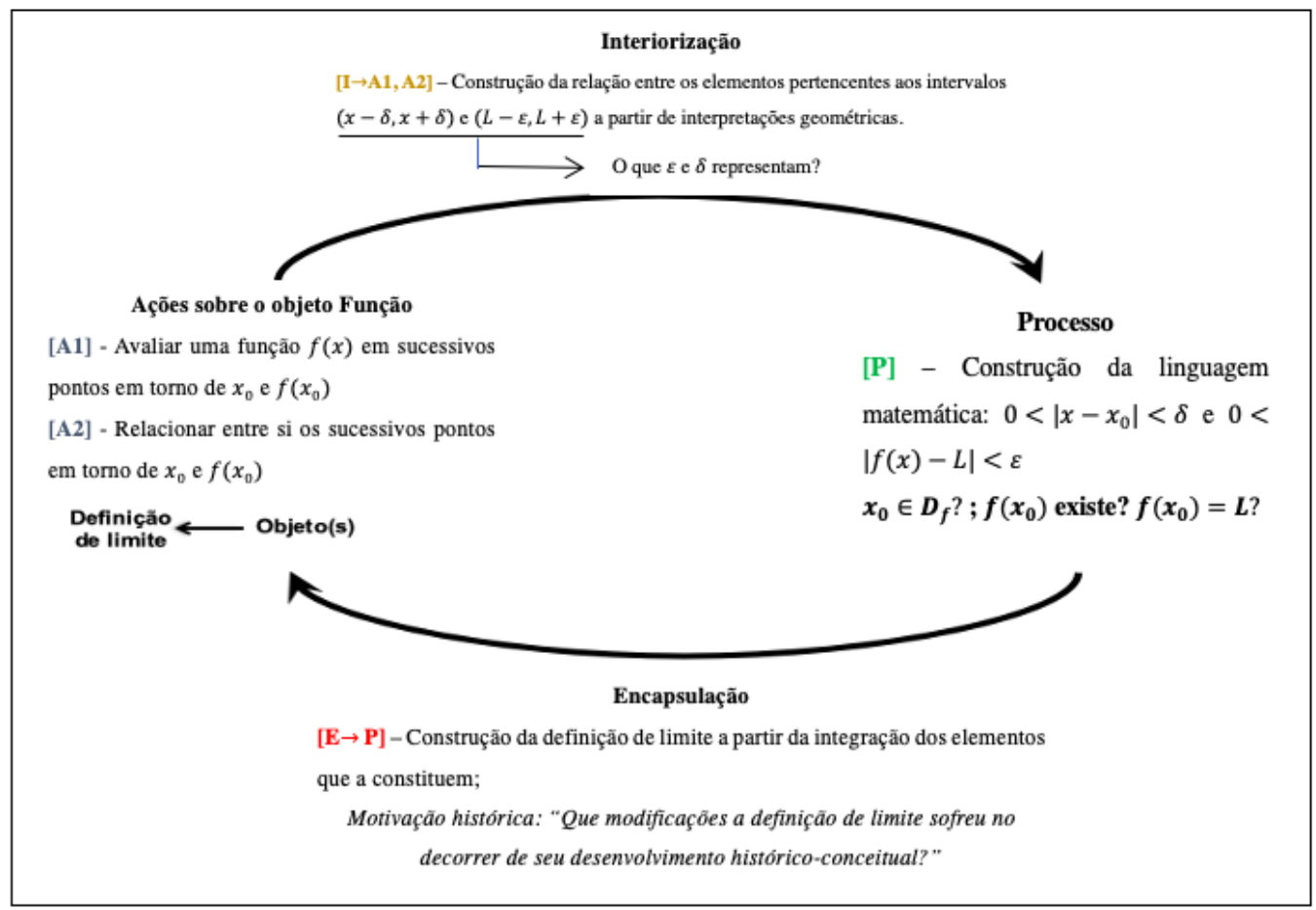

Fonte: Adaptado de Messias (2018, p. 149)

Em [P], admitimos a importância de elaborar uma linguagem matemática mais próxima da definição formal. Desse modo, a partir das construções anteriores, acreditamos ser possível realizar a escrita de $0<\left|x-x_{0}\right|<\delta$ e $0<|f(x)-L|<\varepsilon$, bem como interpretações relativas aos elementos que compõem a definição de limite de uma função. Destacamos, nesse sentido, alguns questionamentos que podem ser levantados nesse momento: " $x_{0}$ deve pertencer ao domínio de $f$ ?"; " $f\left(x_{0}\right)$ precisa existir?"; " $f\left(x_{0}\right)$ é sempre igual a $L ? "$.

$\mathrm{Na}$ encapsulação $[\mathrm{E} \rightarrow \mathrm{P}]$, prevemos a construção da definição de limite a partir da integração dos elementos que as constitui (o termo função, domínio, os intervalos, $\varepsilon, \delta$, etc...). Discussões relativas às modificações que a definição de limite de uma função sofreu no decorrer de seu desenvolvimento histórico podem viabilizar a apreensão desse conceito, de modo a permitir que os estudantes amadureçam matematicamente e, principalmente, que a definição de limite de uma função se constitua como um Objeto.

Para complementar o Esquema 1 (Figura 2) e, com o intuito de promover a formulação de compreensões coerentes sobre o conceito de limite, elaboramos outros quatro esquemas, a saber:

Esquema 2: A relação entre $\varepsilon$ e $\delta$ (Figura 3);

Esquema 3: Relação entre os limites laterais e bilateral (Figura 4);

Esquema 4: Propriedades de limites (Figura 5)

Esquema 5: Limites envolvendo infinito (Figura 6); 
Conjecturamos, desse modo, sobre que construções mentais poderiam ser estabelecidas sobre o objeto definição de limite (previamente construída no Esquema 1). Nesse sentido, entendemos que Ações sobre como determinar Épsilons e Deltas, tanto algebricamente quanto geometricamente, deveriam ser contempladas no segundo Esquema, conforme destacamos na figura 3:

Figura 3 - Esquema 2: Relação entre $\boldsymbol{\varepsilon}$ e $\boldsymbol{\delta}$

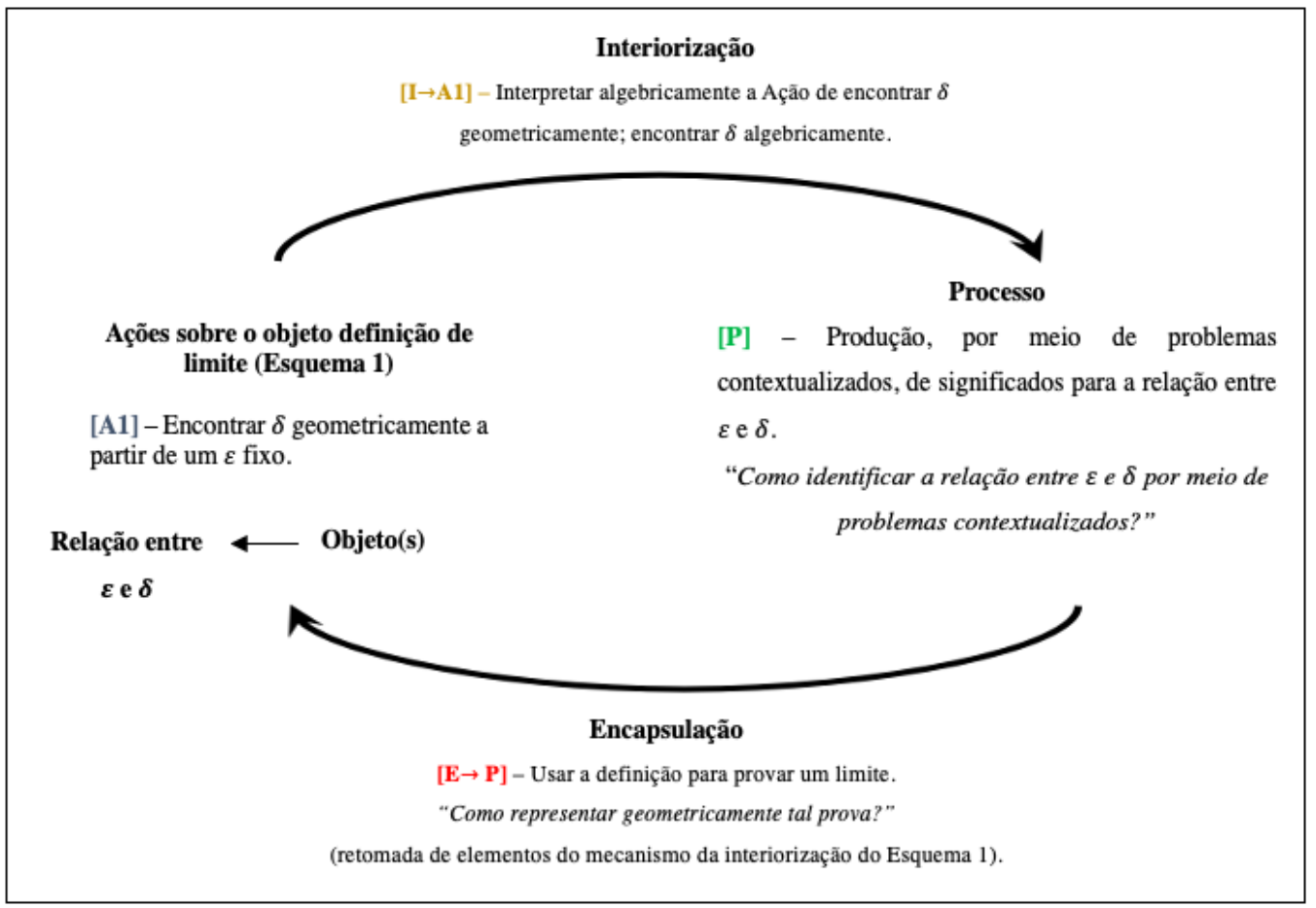

Fonte: Adaptado de Messias (2018, p. 151)

O Esquema 2 é constituído de construções mentais realizadas sobre um objeto prédefinido, nesse caso, a definição de limite, de modo que procuramos enfatizar a relação entre $\varepsilon$ e $\delta$ tanto em termos algébricos quanto em termos geométricos.

A partir da Ação [A1], pretendemos a construção inicial da ideia de que o objeto limite de uma função é norteado por uma relação, entre elementos pertencentes aos intervalos $(L-\varepsilon, L+\varepsilon)$ e $(x-\delta, x+\delta)$, que parte do eixo das ordenadas para o eixo das abscissas (e não o contrário), de modo a auxiliar o indivíduo a distinguir os conceitos de limite de função e função e, principalmente, estender a compreensão da ideia previamente estabelecida por meio do mecanismo da interiorização do Esquema I, isto é, a de que $\mathrm{f}(\mathrm{x})$ permanece em $(\mathrm{L}-\varepsilon, \mathrm{L}+\varepsilon)$, para todos $\mathrm{x} \neq \mathrm{x}_{0}$ pertencente $\mathrm{a}(\mathrm{x}-\delta, \mathrm{x}+\delta)$.

Em [l $\rightarrow A 1]$, admitimos a importância de discutir uma mesma ideia matemática por meio de diferentes representações, de modo a ampliar a compreensão de um indivíduo. Por isso, consideramos coerente interpretar algebricamente [A1], bem como encontrar $\delta$ algebricamente (de forma independente de sua representação geométrica) para que, enfim, o indivíduo passe a ter controle sobre suas Ações sobre o objeto definição de limite, internalizando-as como um Processo que envolve, dentre outros aspectos, a relação entre $\varepsilon$ e $\delta$.

As ações interiorizadas permitem que, a partir de [P], sejam associados significados para a relação entre $\varepsilon$ e $\delta$ por meio de problemas contextualizados que enfatizem não somente tais elementos, mas principalmente, a forma como estes estão relacionados. Mais 
uma vez, é importante vincular essa discussão ao Esquema 1, isto é, ao Objeto definição de limite.

Em $[E \rightarrow P]$, previmos a utilização da definição para provar determinado limite, de maneira a destacar e relacionar entre si todos os elementos que a constituem. Admitimos, mais uma vez, a importância de entender o que tal prova representa geometricamente, por meio da retomada do mecanismo da interiorização do Esquema 1, fato que, a nosso ver, possibilitará uma construção efetiva do Objeto relação entre $\varepsilon$ e $\delta$.

Conjecturamos, também, acerca de construções mentais que pudessem ser estabelecidas, de maneira a possibilitar a apreensão do Objeto relação entre limites laterais e bilateral e, para tanto, construímos o Esquema 3, conforme destacamos na figura 4.

Figura 4 - Esquema 3: Relação entre limites laterais e bilateral

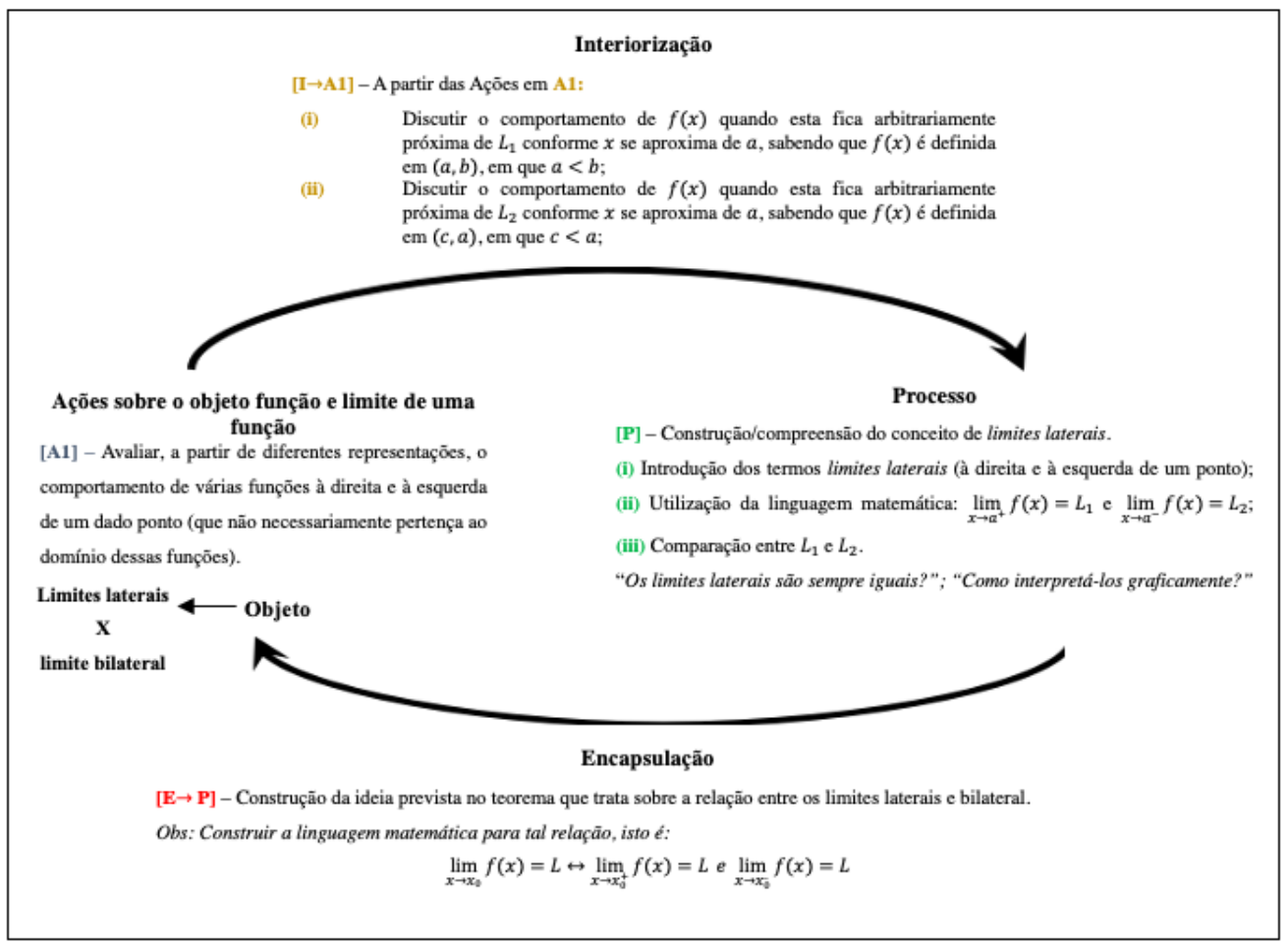

Fonte: Adaptado da Messias (2018, p. 156)

O Esquema 3 foi constituído por construções mentais realizadas sobre o Objeto limite de uma função. Enfatizamos, nesse sentido, a discussão acerca de sua (não) existência, tendo em vista a relação entre os limites laterais e o bilateral.

A Ação [A1] de avaliar o comportamento de diferentes funções (e suas múltiplas representações) à direita e à esquerda de determinado ponto (definido ou não no domínio) foi prevista com intuito de levar o indivíduo a retomar elementos vinculados ao objeto limite de uma função. Nesse caso específico, entendemos que seja importante visualizar que $x_{0} \in(x-\delta, x+\delta)$, que $f(x)$ é definida em $(x-\delta, x+\delta)$, exceto talvez em $x_{0}$. A partir de uma pluralidade de Ações do tipo [A1], é possível generalizar a ideia de que $x-\delta=c$, $x_{0}=a$ e $x+\delta=b$ e, portanto, que $f(x)$ é definida nos intervalos $(c, a)$ e $(a, b)$, sendo $c<a<b$ e $a, b, c \in \mathbb{R}$.

Em [l $\rightarrow A 1]$, admitimos a importância de ampliar a compreensão do indivíduo no que tange ao comportamento de uma função quando esta fica arbitrariamente próxima de: (i) um limite $L_{1}$ à medida que $x$ se aproxima de $a$, sendo $f(x)$ é definida em $(a, b)$ e $a<b$ e 
de (ii) um limite $L_{2}$ à medida que $x$ se aproxima de $a$, sendo $f(x)$ é definida em $(c, a)$ e $c<a$. É possível que, nesse momento, o sujeito perceba que, não necessariamente, $L_{1}=$ $L_{2}$, sendo importante que $[\mathrm{l} \rightarrow \mathrm{A} 1]$ contemple tanto interpretações analíticas quanto geométricas de limites.

Entendemos que a interiorização de [A1] possa levar um indivíduo à construção de [P] e, a partir dele, da compreensão do conceito de limites laterais, de sua notação matemática, ou seja, $\lim _{x \rightarrow x_{0}^{+}} f(x)=L_{1}$ e $\lim _{x \rightarrow x_{0}^{-}} f(x)=L_{2}$ e, especialmente, do fato de que não necessariamente $L_{1}=L_{2}$. A interpretação geométrica da relação entre os limites laterais também pode ser incorporada ao esquema a partir de [P]. Nesse momento, é provável que algumas propriedades de limites sejam, mesmo que inconscientemente, incorporadas à imagem conceitual do sujeito ${ }^{6}$.

Na encapsulação, ou seja, em $[\mathrm{E} \rightarrow \mathrm{P}]$, previmos a construção do teorema que trata da relação entre os limites laterais e a existência do limite bilateral, enfatizando sua unicidade em meio a diferentes representações e sua notação matemática para que, enfim, tal relação alcance o status de Objeto na mente de um indivíduo.

Figura 5 - Esquema 4: Propriedades de Limite

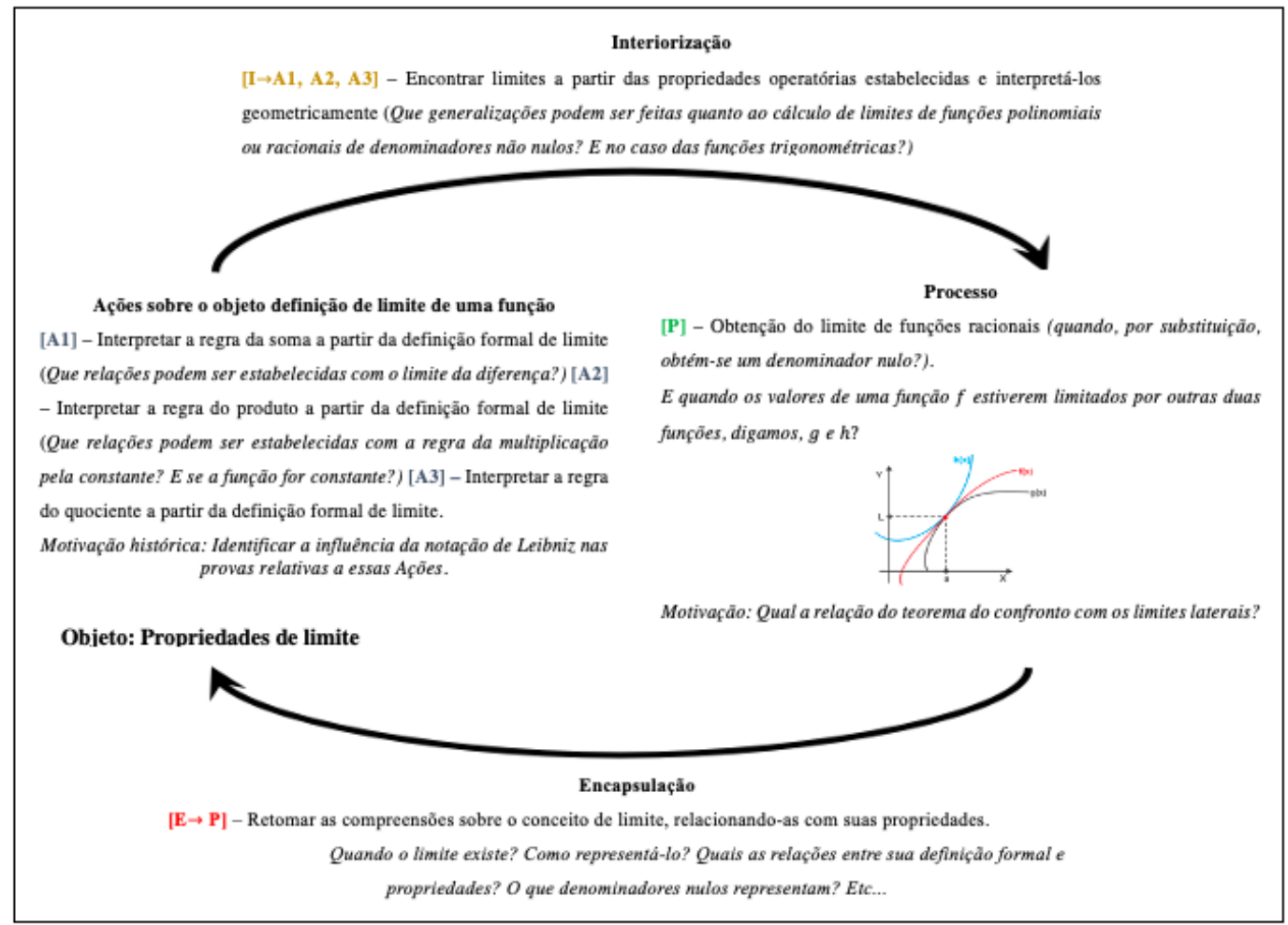

Fonte: Adaptado de Messias (2018, p. 159)

Tendo a definição de limite, a relação entre $\varepsilon$ e $\delta$ e entre os limites laterais e o bilateral como objetos pré-definidos, é possível avançar para a construção de um esquema que

\footnotetext{
${ }^{6}$ Dedicamos o esquema 4 à construção de mecanismos e processos mentais vinculados a tais propriedades. Nesse sentido, evitaríamos que a generalização equivocada de práticas operatórias fosse atrelada a interpretações sobre a natureza do conceito de limite.
} 
contemple as propriedades inerentes a esse conceito, tais como os teoremas que tratam das regras operatórias do limite, do cálculo de limite de funções polinomiais e de funções racionais (com denominadores não nulos), o teorema do confronto (e suas aplicações), limites envolvendo funções trigonométricas, dentre outras situações, as quais apresentamos no esquema 4.

No Esquema 4 foram contempladas construções mentais relacionadas ao Objeto propriedades de limite. Enfatizamos, nesse sentido, a discussão acerca de regras operatórias a partir de conhecimentos previamente estabelecidos.

As Ações [A1], [A2] e [A3] foram relacionadas às regras operatórias de limite. Nesse sentido, previmos relacioná-las com a definição de limite (previamente construída em esquemas anteriores). Entendemos que o indivíduo precisa compreender, por exemplo, o porquê de $\lim _{x \rightarrow x_{0}}(f(x)+g(x))=\lim _{x \rightarrow x_{0}} f(x)+\lim _{x \rightarrow x_{0}} g(x)=L+M$, sendo $L, M \in \mathbb{R}$, e não simplesmente memorizá-la de forma desconectada dos elementos matemáticos vinculados a esse conceito (fato que contribui para a lacuna entre teoria formal e práticas operatórias envolvendo cálculo de limites). Como motivação, pensamos em relacionar as referidas Ações ao Cálculo de Leibniz, especialmente no que tange a notação matemática utilizada.

Consideramos importante que a interiorização das Ações, isto é, [l $\rightarrow A 1, A 2, A 3]$, leve o sujeito a encontrar limites (inclusive de funções trigonométricas e de funções definidas em mais de uma sentença) a partir das propriedades operatórias estabelecidas, interpretandoos geometricamente. A partir dessas práticas, é possível buscar a generalização no que se refere ao cálculo de limite de funções polinomiais e racionais (que não resultem em denominadores nulos), uma vez que ambos podem ser calculados por substituição. Nesse sentido, é importante reiterar (por meio das atividades propostas) que nem sempre $\lim _{x \rightarrow x_{0}} f(x)=f\left(x_{0}\right)$ e, portanto, é preciso entender que limite e função não são um mesmo objeto matemático.

[P] traz consigo a ênfase em duas situações que podem ser associadas às ações interiorizadas: o cálculo de limite de funções racionais (que implique na emergência de denominador nulo) e a interpretação do teorema do confronto, enquanto alternativa para encontrar o limite de uma função $f(x)$ que seja limitada por outras duas funções, digamos, $g(x)$ e $h(x)$. Nosso intuito é o de ampliar a compreensão sobre limite e não somente condicioná-lo à mera substituição de valores de $x$, ou à prática de eliminação de denominadores nulos. Admitimos, nesse sentido, a importância de levar um indivíduo a entender o que uma indeterminação pode representar nesse contexto. Entendemos que traçar conexões entre tais situações e a representação algébrica e geométrica de limites laterais seja uma boa estratégia para expandir as situações matemáticas que podem ser vinculadas a esse conceito.

Na encapsulação, ou seja, em $[\mathrm{E} \rightarrow \mathrm{P}]$, propusemos a retomada de elementos previstos nos esquemas anteriores, por meio de reflexões acerca da existência do limite, de sua relação com limites laterais, com práticas operatórias utilizadas para calcular limites de diferentes funções, dentre outros. Conjecturamos que, dessa maneira, seja possível promover a construção do Objeto propriedades de limite, bem como de expandir os domínios de validade desse conceito por meio da generalização e abstração dos elementos que o constituem, tendo em vista uma pluralidade de contextos e múltiplas representações. 
Finalmente, a fim de complementar a decomposição genética, elaboramos o esquema 5 que, por sua vez, foi constituído de elementos vinculados à construção do objeto limite envolvendo infinito, conforme destacamos na figura 6.

Figura 6 - Esquema 5: Limites envolvendo infinito

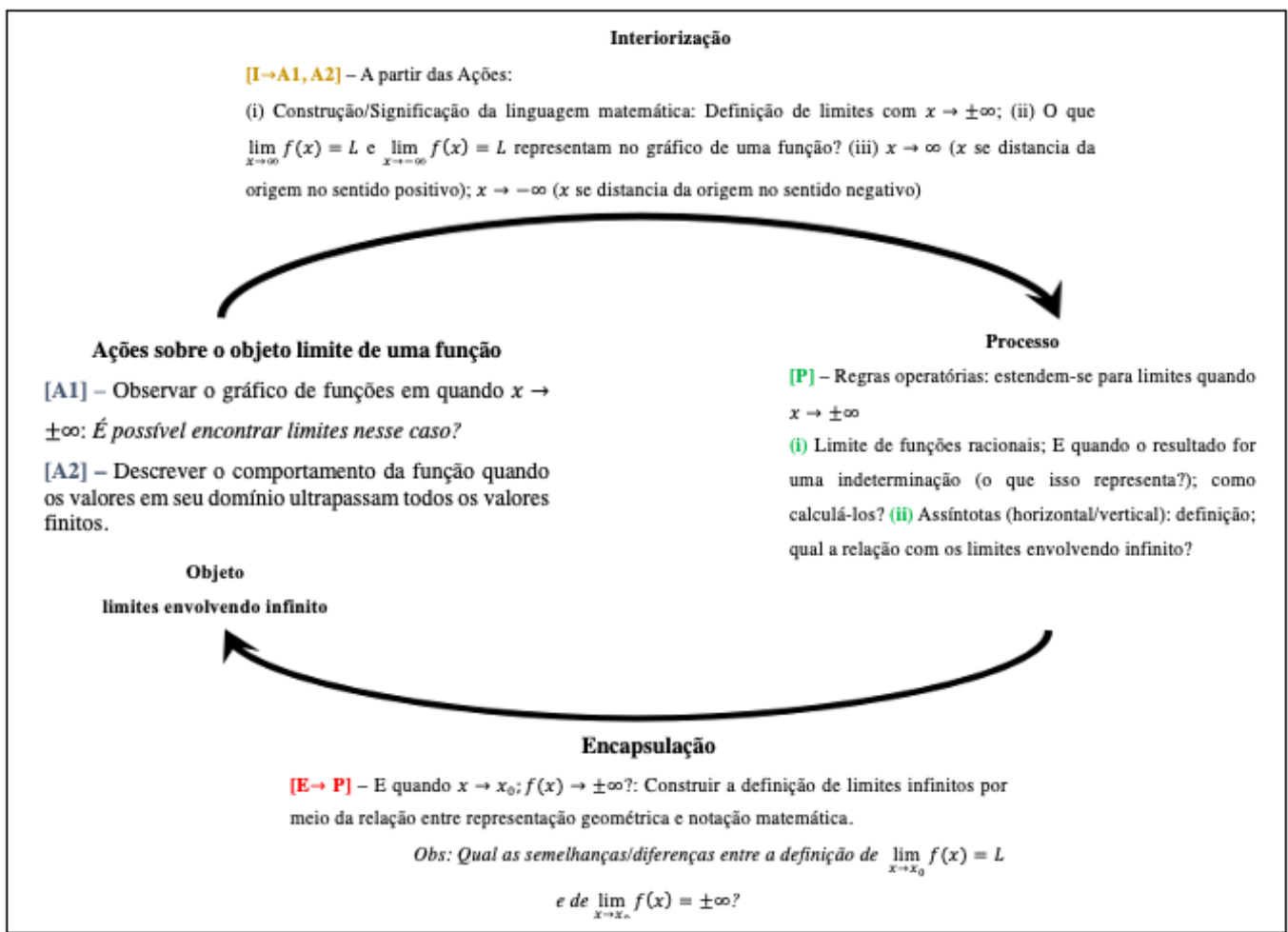

Fonte: Adaptado de Messias (2018, p. 162)

Conjecturamos, no Esquema 5, sobre construções mentais que pudessem viabilizar a apreensão do Objeto limites envolvendo infinito. Enfatizamos, nesse sentido, discussões sobre o que $\lim _{x \rightarrow x_{0}} f(x)= \pm \infty$ e $\lim _{x \rightarrow \pm \infty} f(x)=L$ significam, sua relação com outros conceitos, como o de assíntotas vertical e horizontal, sua representação geométrica, dentre outros aspectos.

As Ações [A1] e [A2] foram relacionadas à observação do comportamento de funções quando $x \rightarrow \pm \infty$, de modo a avaliar o que acontece quando os valores do domínio se afastam da origem nos sentidos positivo e/ou negativo. Nossa expectativa é a de que, por meio dessas ações, o indivíduo possa construir certa compreensão acerca da ideia de $x \rightarrow \pm \infty$ e, especialmente, de como tal comportamento pode ser visualizado, tendo em vista a representação gráfica de diferentes funções.

Consideramos fundamental que o mecanismo de interiorização das Ações, ou seja, [l $\rightarrow \mathrm{A} 1, \mathrm{~A} 2]$ leve $\mathrm{o}$ indivíduo a construir a definição de limites quando $x \rightarrow \pm \infty$, de maneira a compreender a ideia de $\lim _{x \rightarrow \pm \infty} f(x)=L$, tanto em termos algébricos quanto geométricos. Nesse sentido, admitimos a importância de vincular a tais situações a ideia de que, à medida que $x$ se distanciar da origem (no sentido positivo ou negativo), $f(x) \rightarrow L$.

Nossa expectativa para [P] é a de que o indivíduo consiga estender, a partir do que fora construído por meio do mecanismo da interiorização, as regras operatórias (estabelecidas previamente no Esquema 4) para o cálculo de limites envolvendo o infinito, bem como o cálculo e interpretação de limites de funções racionais quando $x \rightarrow \pm \infty$ 
(inclusive no caso de emergência de indeterminações). Previmos, também, a construção da definição e representação de assíntotas (horizontais e verticais), de maneira a estabelecer a relação com os limites quando $x \rightarrow \pm \infty$ e com a ideia de limites infinitos, cuja definição foi incluída como um elemento constituinte do mecanismo da encapsulação.

Na encapsulação, isto é, $[\mathrm{E} \rightarrow \mathrm{P}]$, propusemos a construção da definição de limites infinitos a partir da relação entre sua representação geométrica e notação matemática, de maneira semelhante à forma como a definição de limite foi construída no Esquema 1. Admitimos, ainda, a importância de comparar as definições de $\lim _{\mathrm{x} \rightarrow \mathrm{x}_{0}} \mathrm{f}(\mathrm{x})=\mathrm{L}$ e de $\lim _{\mathrm{x} \rightarrow \mathrm{x}_{0}} \mathrm{f}(\mathrm{x})= \pm \infty$, tendo em vista que muitos estudantes não costumam fazer distinção entre ambas, conforme evidenciamos, inclusive, em nosso estudo preliminar. Conjecturamos que, dessa maneira, seja possível apreender o objeto limites envolvendo infinito em meio a múltiplos contextos e representações.

A Decomposição Genética para o objeto Limite de Função construída foi composta pelos cinco esquemas apresentados nas figuras 2 a 6, conforme destacamos na figura 7 .

Figura 7 - Decomposição Genética para o conceito de limite

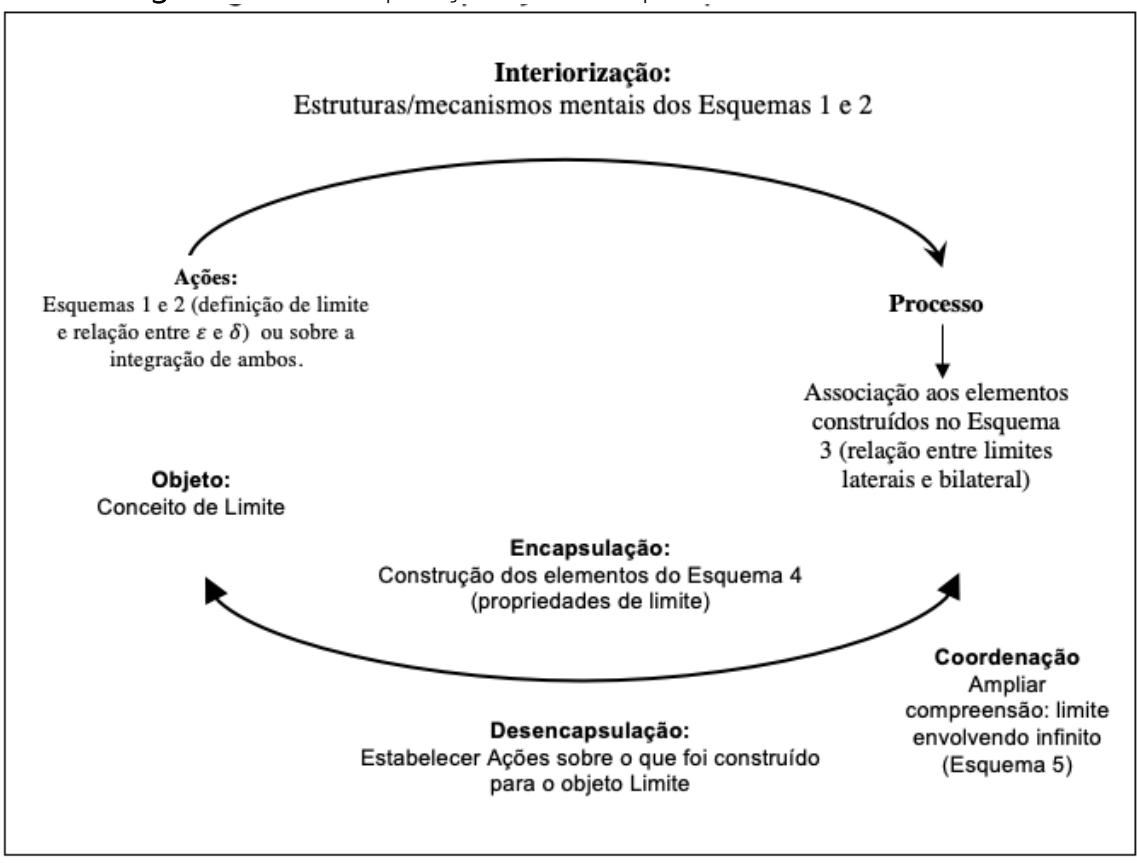

Fonte: Messias (2018, p.164)

Ressaltamos que essa decomposição genética, enquanto modelo de epistemologia e cognição matemática, foi elaborada tendo em vista nossas experiências docentes, os apontamentos da literatura sobre a compreensão de estudantes acerca do conceito de limite, o aprofundamento teórico relativo à teoria APOS. Incluímos, ainda, alguns aspectos concernentes ao desenvolvimento histórico dos referidos conceitos.

Reiteramos, finalmente, a relevância desse estudo, por meio do qual foi possível traçarmos reflexões e conjecturas sobre os elementos que, a nosso ver, precisam ser vinculados ao processo de apreensão do conceito de limite de uma função, de modo a possibilitar uma aprendizagem efetiva desses conhecimentos em uma pluralidade de contextos matemáticos. 


\section{Considerações finais}

Contemplamos, nesse trabalho, nossas considerações relativas a um estudo, cujo objetivo foi conjecturar sobre que estruturas e mecanismos mentais precisam ser construídos por um indivíduo de modo a possibilitá-lo compreender efetivamente o conceito de limite. Norteamo-nos, para tanto, na seguinte questão: "Que estruturas e mecanismos mentais precisam ser construídos de modo a permitir que estudantes compreendam o conceito de limite de uma função?".

Para responder a essa pergunta, elaboramos uma decomposição genética para o conceito de limite de uma função. Por meio desse modelo, traçamos reflexões sobre uma pluralidade de construções mentais as quais assumimos ser de grande importância para que um indivíduo compreenda efetivamente tais objetos. Baseamo-nos, para tanto, nos apontamentos da teoria APOS, bem como nas compreensões elencadas ao longo desse trabalho.

A Decomposição Genética foi constituída de cinco esquemas, por meio dos quais previmos a construção dos Objetos definição de limite, relação entre $\varepsilon$ e $\delta$, relação entre limites laterais e bilateral, propriedades de limite e limites envolvendo infinito, incorporandoos, em seguida, a uma única estrutura mental que, por sua vez, admitimos poder levar um indivíduo à apreensão do objeto conceito de limite de uma função.

Reiteramos, nesse sentido, que esse trabalho contém importantes contribuições para futuros estudos sobre o tema abordado, bem como para a elaboração de instrumentos avaliativos e materiais instrucionais sobre a aprendizagem do conceito de limite. Ressaltamos, ainda, a importância de ter trazido para discussão elementos concernentes à teoria APOS, fato que pode contribuir para a expansão de pesquisas que contemplem essa perspectiva teórica e articulem-na à aprendizagem de diferentes conceitos matemáticos.

Salientamos, por fim, a relevância desse trabalho no que tange aos estudos efetivados no âmbito da Educação Matemática, especialmente, em se tratando da compreensão do conceito de limite de uma função, o qual é de grande importância para a aprendizagem de outros conceitos pertencentes à esfera de conhecimentos do Cálculo.

\section{Referências}

AMATANGELO, Miriam Lynne. Student understanding of limit and continuity at a point: a look at four potentially problematic conceptions. 2013. 112f. Dissertação (Mestrado em Artes), Bringham Young University (Utah/USA), 2013.

ARNON et al. APOS Theory - a framework for research and curriculum development in mathematics education. New York: Springer, 2014

CORNU, B. Apprentissage de la notion de limite - conceptions et obstacles. Tese de doutorado (matemática). Université Scientifique et Medicale de Grenoble, 1983.

CORNU, B. Limits. In: Advanced Mathematical Thinking (ed. David Tall). Kluwer publications, 1991.

DENBEL, D.G. Students misconceptions of the limit concept in a first Calculus course. Journal of Education and Practice, v.5, n³4, 2014. 
JORDAAN, T. Misconceptions of the limit concept in a mathematics course for engineering students. Dissertação de mestrado (educação matemática). University of South Africa, 2005.

HITT, F.; LARA-CHAVEZ, H. Limits, continuity and discontinuity of functions from two points of view: that of the teachers and that of the students. In: BILLS, L (ED). Proceedings of the British Society for Research into Learning Mathematics, v. 19, p. 49 - 54, jun.1999.

JUTER, K. Learning limits of function: students' conceptual development. Saarbrücken: VDM Verlag Dr. Müller, 2008

KARATAS et al. A cross-age study of students' understanding of limit and continuity concepts. BOLEMA, Rio Claro (SP), v. 24, n 38, p. 245 a 264, abril, 2011.

MESSIAS, M. A. V. F. Teorias Cognitivas do Pensamento Matemático Avançado e o processo de construção do conhecimento: um estudo envolvendo os conceitos de limite e continuidade. 2018. 186f. Tese (Doutorado em Educação em Ciências e Matemáticas), Programa de Pós-Graduação em Educação em Ciências e Matemáticas, UFPA, 2018.

MESSIAS, M. A. V. F; BRANDEMBERG, J. C. Discussões sobre a relação entre limite e continuidade de uma função: investigando imagens conceituais. BOLEMA, Rio Claro (SP), v. 29, n.53, p. 1224 - 1241, dez., 2015.

PRZENIOSLO, M. Images of the limit of function formed in the course of mathematical studies in the university. Educational Studies in Mathematics, Netherlands, v. 55, p. $103-132$, 2004.

ROA-FUENTES, S; OKTAÇ, A. Construcción de una decomposición genética: Análisis teórico del concepto transformación lineal. Revista Latino-americana de Investigación Matemática Educativa, n.13, v.1, p. 89-112, 2010.

SARVESTANI, A.K. Contemplating problems taken from history of limits as a way to improve students' understanding of the limit concept. 2011. 162f. Tese de Doutorado, Universiteit Van Amsterdam, 2011.

TALL, D; VINNER, S. Concept image and concept definition with particular reference to limits and continuity. Educational Studies in Mathematics, n. 12, 1981, p. $151-169$. 